\title{
Is Lean Management implementable in a department of radiology?
}

\author{
Jens Karstoft $\cdot$ Lene Tarp
}

Received: 7 June 2010 / Accepted: 23 September 2010 / Published online: 22 March 2011

(C) The Author(s) 2011. This article is published with open access at Springerlink.com

\begin{abstract}
Objectives Due to an increasing demand for more examinations in a non-profitmaking hospital with a fixed budget, Lean Management was introduced in the Department of Radiology in 2006. The process, experience and results are discussed from a management view.

Methods Lean principles were introduced in the department and ways of working were adjusted accordingly.

Results Higher productivity and shorter waiting lists were achieved, along with extra time for education of the staff. Conclusions Lean Management can successfully be used in a hospital radiology department. However, introducing Lean Management caused resistance from especially the mid-level managers.
\end{abstract}

Keywords Radiology · Organizational productivity . Lean management

\section{Introduction}

The Lean concept, initially called The Toyota Production System, was introduced around 1950 because of low efficiency at The Toyota Motor Company. The Production system was developed after visiting the Ford Company in Detroit, and thus partly inspired by Western Taylorism and assembly line production at the Ford Company [1, 2]. However, The Toyota Production system differed in many ways from the established way of leading production. It was not just another tool to improve output in the production line. It implied a change in

J. Karstoft $(\bowtie) \cdot$ L. Tarp

Department of Radiology, Odense University Hospital,

5000 Odense, C, Denmark

e-mail: Jens.karstoft@ouh.regionsyddanmark.dk working culture. First, it was employee-driven with continuous improvements, called "kaizen". Second, it focused on keeping up the flow in all steps of the production and minimising all kind of waste in each step. Third, it focused on what was of value to the customers and eliminated procedures that did not give value to the customer. The Toyota Production system was optimised in the following decades and in the 1990s introduced into western industries as the Lean Concept [3].

Initially Lean was popular within production companies, but during recent years many public organisations have also become highly interested in the method, and it has been introduced to the healthcare system as well. But is it possible to use a production system from industry in the hospital sector? A system on a large scale trying to standardise and depersonalise production, taking away the normal personal variance in how we do things. How could that possibly be used in diagnosing and treating the great variety of human diseases?

Odense University Hospital (OUH) is one of the largest hospitals in Denmark, covering 10\% of the Danish healthcare system. On a yearly basis, the number of in-patients is 105,000 and the number of out-patient visits is 900,000 . The yearly budget is 700-million euros. Being a public government financed hospital with limited or no finance from treating extra patients, OUH is constantly under economic pressure. A public hospital is an operating organisation with a tight budget and high production. The organisation is customer-minded, and orientated towards development and innovation. The Department of Radiology has a budget of 18-million euros, 200 employees and 40 radiography/medical students. Two-hundred-thousand examinations/treatments were carried out in 2009, and all kinds of radiology procedures are performed.

The radiologists are organised in relation to their clinical specialty, and the radiographers and secretaries into five 
teams with specialised functions in magnetic resonance imaging (MRI), computed tomography (CT), digital radiography (DR) $\mathrm{CT}$, neuro intervention, and angio intervention.

\section{Background}

The reason for OUH to start working with Lean was partly due to an increasing demand for more examinations and treatments at the hospital without increasing costs-and partly as a coincidence. In 2005 the general manager from a large private industrial company had been hospitalised in a department at OUH. The private company had been under pressure from companies in other parts of the world with lower salary costs and thus lower prices of comparable products. A few years earlier, the manager had therefore introduced Lean with very good results. During his stay at the hospital, he observed the way the staff worked and thought there was much wasted time, inadequate practices and lack of visual communication. Subsequently there was a dialogue between the manager of the large industrial company and the manager of OUH, where they discussed Lean and efficiency. The result was a collaboration and coaching between the private enterprise and the hospital in implementing Lean at the hospital.

That OUH should start working with Lean was a top-down decision. Along with two other departments, Cardiology and the Orthopaedic Department, the Radiology Department was chosen to be part of a pilot project for implementation of Lean at the hospital. To initialise the process, the board of directors of OUH had engaged a private consulting company with Lean experience. Companies like these were becoming more and more common since Lean had become "the buzz word" in modern management in industry. Meetings were held with the heads of the three departments together with the private Lean consultancy company. As heads of the departments, we had what one might call a natural scepticism towards introducing Lean into our departments. However, the board of directors had a willingness to take the risk, as regards the economy, the organisation and the working environment. Furthermore, it was stated that $55 \%$ of what could be gained in production due to implementing Lean would pass on to the departments. Consequently, during the summer of 2006, the three departments started working with Lean without any former experience and only theoretical knowledge of the Lean concept.

\section{The concerns}

The heterogeneity of production

The Radiology Department carries out all kinds of radiological examinations. In all, there are approximately 550 different procedures in the radiology information system (RIS). Both in- and outpatients are examined. Approximately $50 \%$ of the examinations are referred as acute cases. Patients are referred from both inside the Hospital and by general practitioners. This is quite heterogeneous production compared with that of the industrial companies for which Lean was invented. It would be difficult to standardise our procedures. It did not seem possible to set up a uniform day production program; for instance, carrying out only CT of the head one day, and CT of the thorax the next.

\section{Resistance to change}

As Lean is Japanese, many of the words used were totally unfamiliar to the employees. This in itself could lead to resistance to the Lean concept. Therefore, it was important to find ways to de-dramatise Lean during the introductory period. One way was using lots of information mixed with cartoons describing inappropriate ways of doing things.

How to encourage the staff

Lean is supposed to be employee-driven. But how could we get the employees engaged in the concept and simultaneously bring up ideas of how to increase productivity? The director stated that $55 \%$ of the gains obtained by working with Lean would go to the departments. As OUH is a nonprofit-making hospital, we decided that increased productivity should free up time for the staff to do other activities. This meant that if Lean could increase production, only $45 \%$ of this output should go towards more examinations, and $55 \%$ should go towards other activities, i.e. education, courses, conferences, etc. Actually, with the effect of Lean, it became quite a task for the employees to come up with ideas about what the potential spare time should be used for. Looking back, only a small percentage of the extra outcome based on the Lean process has been used by the employees of the department. However, as Lean has to be employee-driven, it seemed a good idea for the process to benefit the employees. Otherwise it would be difficult for them to get enthusiastic and thus come up with good ideas about how to maximise production. Even though the slogan for Lean is "work smarter, not harder" it did not seem realistic for the staff to do this without a goal or benefit for themselves. The motive for inventing Lean in the Toyota Company and introducing it into the Danish private company mentioned above was a crisis threatening the existence of the company and thereby the jobs and welfare of the employees, a situation that will be motivating for everybody to think up ideas about how to increase production and eventually get an increased salary. However, in a non-profit institution such as $\mathrm{OUH}$, in a radiology department with a constant lack of radiographers and 
radiologists, there was no threat to jobs and thus no incentive to come up with ideas about how to increase production. Nor could we tempt the staff with a raise in the salary as OUH is a non-profit-making hospital and the salaries are largely negotiated centrally. Under these circumstances, the incentive had to be a different one and therefore we proposed to increase the time spent on education, conferences, research, etc. Indeed, we even proposed that people could go jogging on Wednesday mornings in Lean-gained spare time. This could be a way of showing the opponents, the doubters and the other departments that Lean was having an effect, and that the 55\% benefit was going to be taken seriously. Unfortunately no one seemed interested and we were unable to implement the idea.

\section{The process and some of the results}

To initialise the process, the department had support from a consult - a production engineer from a private consulting firm for approximately 20 hours a week. It was a collision of two different worlds. However, it soon became apparent that the cooperation was valuable. The role of the consult was especially to ask us and the staff the critical "hows" and "whys" regarding our production, questions that only a production-minded person from outside the institution with a non-radiological background would ask. During the summer of 2006 we decided to establish a Lean group in the department, and two members of the group completed a certified Lean training course from the consulting firm. The heads of the department were working closely with the Lean group, and were actively participating and being visible in the process. There was no new capital investment in the budget, thus it was necessary to collect all resources from within the department and to prioritise Lean.

The strategy was then to make broad information about Lean available to all the employees and to go through each section one by one.

The Lean group:

- One full-time team leader

- One secretary, 3 days a week

- One superintendent, 3 days a week

- One radiologist, 1 day a week

- One economist, ad hoc

- Ad hoc assistance from a consulting firm

Who the leader of the team was turned out to be crucial. The person would become the representative of the Lean concept. In the Radiology Department, we had employed our own economists for approximately 5 years. With his theoretical background, the economist was initially appointed to be the team leader. However, it was clear that the vast knowledge of radiological procedures and professional respect as well as the skills to inspire the employees seemed to be the crucial tasks for the team leader, rather than understanding the theoretical Lean concept or making estimates of the economic effects. Therefore, the team leader was replaced with a well-known and clinically respected radiographer. All members of the group started from scratch without any previous knowledge of Lean. This meant that they had to present the process to all the employees, arrange theme days, take care of informing and implementing the Lean concept in all the different sections of the department, in addition to educating themselves in Lean management.

Applying Lean to the Radiology Department Lean is what creates value for the customer-how to make flow and remove waste, and to make improvement. The basic Lean concept is based on the five Lean principles and the seven wastes [3]. We tried to apply the industrial production terms to our healthcare service in the following way.

The five Lean principles:

1. Specify what creates value for the customer - to see our production from the patients' point of view

2. Identify all the steps in the value stream and eliminate the steps that do not create value for the customer-going through the whole process from referral to the final report was very valuable, and an eye-opener for many staff

3. Make the processes flow smoothly-e.g. eliminate piles of reports waiting to be signed and other inappropriate ways of working

4. Let the needs of the customer lead the processexamination times convenient to the patient and/or referral unit

5. Strive for perfection by continually removing waste

The seven wastes:

1. Overproduction - examinations that are unnecessary or too early

2. Idle time-waiting for patients, colleagues or materials

3. Operator motions - unnecessary movements because of bad application

4. Processing - unnecessary processes that compensate for weaknesses elsewhere

5. Inventory - ties up capital, space and time; materials go out of date

6. Transport-moving materials, patients or papers over long distances

7. Defects - waste of time, resources and materials

Results-Lean in the CT section One of the first projects was in the CT section. The reasons for starting in this section were:

- Waiting time for CT examinations and reports 
- Many acute examinations made it difficult to get a comprehensive view of the day's program

- The staff was always behind with the booking schedule

- Many interruptions, changes and noise resulting in a bad working environment

- Lack of time for staff training/education and development

The basic tools used:

Kaizen The word "kaizen" means continuous improvement. To initiate the process and make it visible, a whiteboard was set up in the CT section, where everyone could place a note with a problem or a suggestion for improving a procedure. Once a week, a group from the CT team - physicians, radiographers, secretaries and leadersheld a 15-min meeting discussing the suggestions and agreeing how to implement them.

VSM (value stream mapping) A member of the Lean group, together with a member from the CT team, follow a patient through the current system from arrival from the referring physician until the report is sent back. They measure the time taken for every step in the workflow and the waiting time between the steps. Each step is analysed and they map out what creates value for the patient. They identify problems in the workflow, make an effort to solve the problems, and make a new presentation of the future flow. We have waiting lists for patients, except for acute and cancer patients. Overall, the run-through time for the patients with the longest waiting times were 3 months from start to finish, of which only $133 \mathrm{~min}$ were of value to the patient — a shocking result for everybody in the section. Having worked through this problem, the result of the group's great efforts decreased the total run-through time for these patients to 4 weeks.

Five $S$ 's The five S's constitute a relatively simple, rapid, low-cost and low-tech way of finding more time for the staff (Fig. 1).

- Sort - get rid of unused materials

- Set in order-get organised

- Shine-keep work areas clean

- Standardise - establish standards to keep areas clean and organised

- Sustain — establish procedures to prevent backsliding

SMED (single-minute exchange of die) SMED is used to reduce changeover time between the patients. The examination time must be separated into internal and external steps. Internal steps must be carried out on the CT system, and the external steps can be carried out elsewhere. We have found out that the total examination time was too long compared with the imaging time.

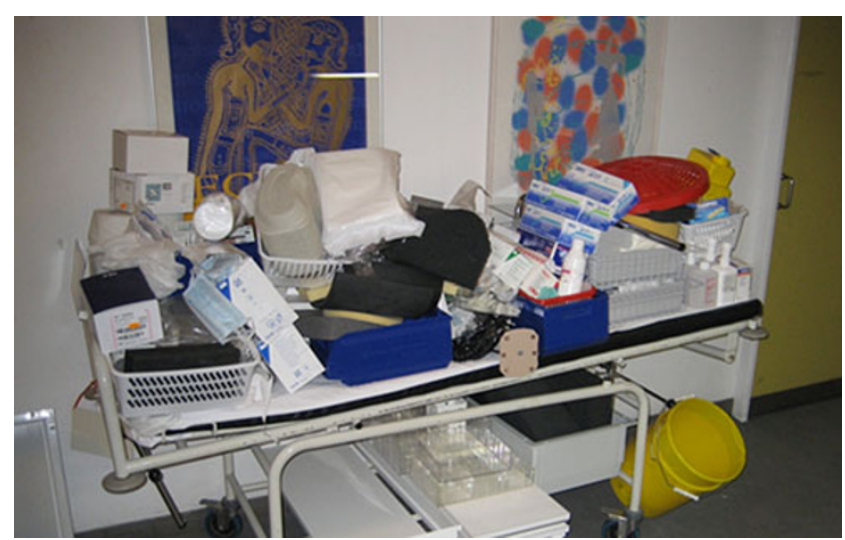

Fig. 1 "Left-overs" after applying the five S's to the CT rooms

To find the reason for this we filmed a number of examinations and found out that we were spending a lot of time informing the patient and inserting a cannula with the patient lying in the CT device.

One of the solutions was to establish a preparation room.

Now a radiographer takes in the first patient-prepares $\mathrm{him} /$ her for imaging outside the CT room and then the same radiographer takes the patient in to carry out imaging. Meanwhile, another radiographer takes the next patient into the preparation room and so on. This gives up to $50 \%$ reduction in in-room time.

Outcome in the CT section With better planning of examination programmes, protocols, etc., we gained enough time to examine six more patients during the daytime. Over a year, this translates into about 900 patients. The total effect in number of examinations is shown in Fig. 2. Consequently, the waiting lists fell dramatically (Fig. 2), an effect we found in all of the sections after we had introduced Lean.

As it had been decided that the staff should have $55 \%$ of the gains of the better organisation, they got opportunities to plan educational and theme days. However, as was stated above, only a small percentage of the gain was actually used by the staff.

The response from the staff was positive - they had an easier overview of the day's programme, a better workflow/ environment, and became focused on continuing improvement.

Similar effects were seen in most of the other sections in the department. Figure 3 shows the waiting list during the period when Lean was implemented in the section, as well as the current waiting list.

\section{Lean at the Radiology Department today}

After half a year the cooperation with the production engineer was gradually reduced. Lean has become a natural 
Fig. 2 Effects of Lean in the $\mathrm{CT}$ section. Increasing number of examinations and a drop in waiting time

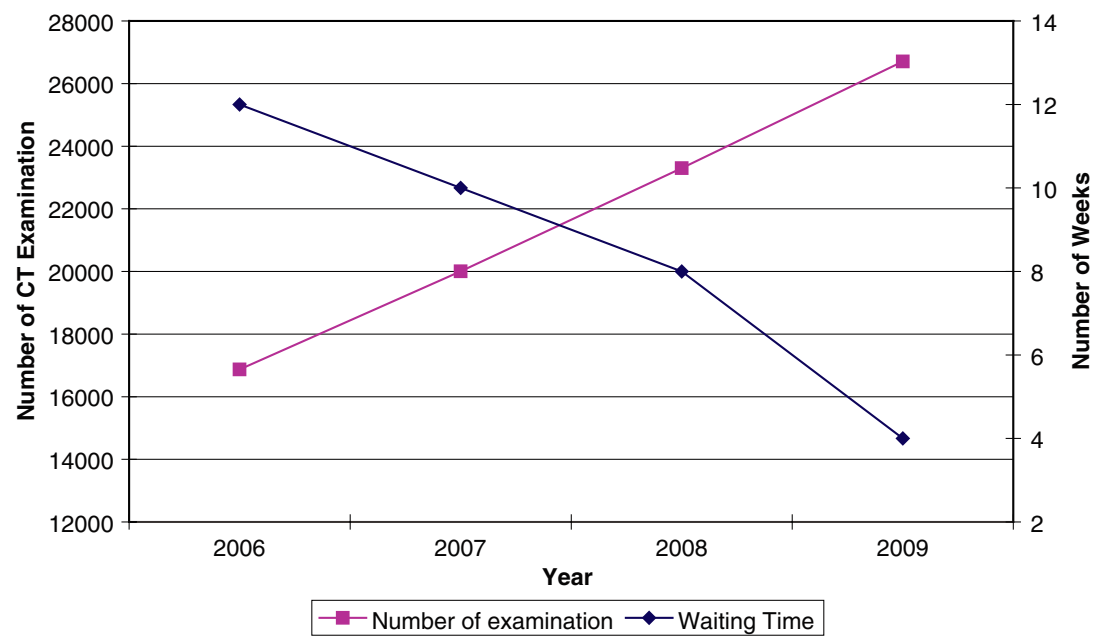

part of our department's production. There was a risk that the Lean way of working would cease and the staff would go back to the old, more conservative ways of doing things. Therefore the Lean working method was adjusted to the world of the Radiology Department. For instance we have made our own "extended kaizen boards" and every Monday 15-min extended kaizen meetings are held with each team in the whole department. At the meetings all members of the team participate as well as the heads of the department. An example is shown in Fig. 4.

Production:

- MRI referrals received per day during the last week

- MRI examinations per day-for each section: oncology, musculoskeletal, neuro, abdominal

- Referrals waiting to be booked

- Referrals waiting to be prioritised

- Waiting time for anaesthesia

- Examinations not reported within $24 \mathrm{~h}$

Goals:

- Two from the heads of the department

- Two from the team

- Possible problems

- Evaluation: what is the status of the different goals?
- Improvements: different problems, proposals, good ideas from the whole staff.

- Action plan: who does what?

Decision-making is easier as the competences are gathered and often there is no need for subsequent meetings, as the tasks are distributed at the 15-min target meetings. The decision method and time to action have been reduced.

\section{Discussion}

Denmark has a tradition for industrial democracy. Codetermination and the workforce have an influence on how to plan the work increase in productivity and job satisfaction [4]. In this respect, part of the Lean concept was natural to introduce, as it had to be employee-driven. However, working with Lean was in many respects a new world to the department. It was one thing to understand the theoretical concept and to learn how to use the different methods, but the true challenge was to manage the change in culture.

As Lean was implemented and rolled out in the department, it soon became apparent that some of the staff
Fig. 3 Effect of Lean in the ultrasound section. Waiting times in weeks for different types of non-acute patients

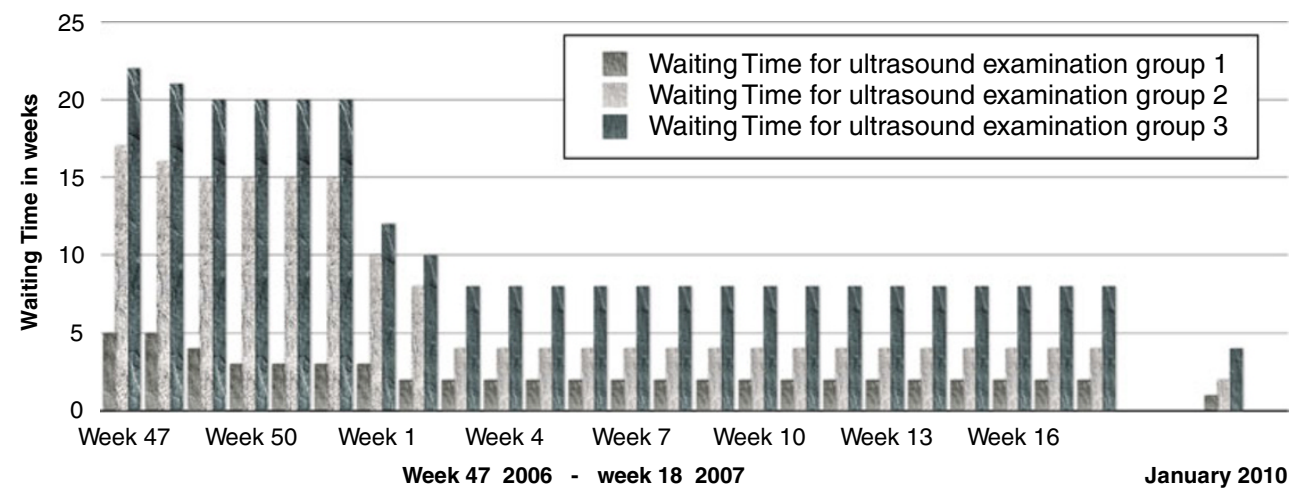


Fig. 4 Extended kaizen meeting with the MRI team

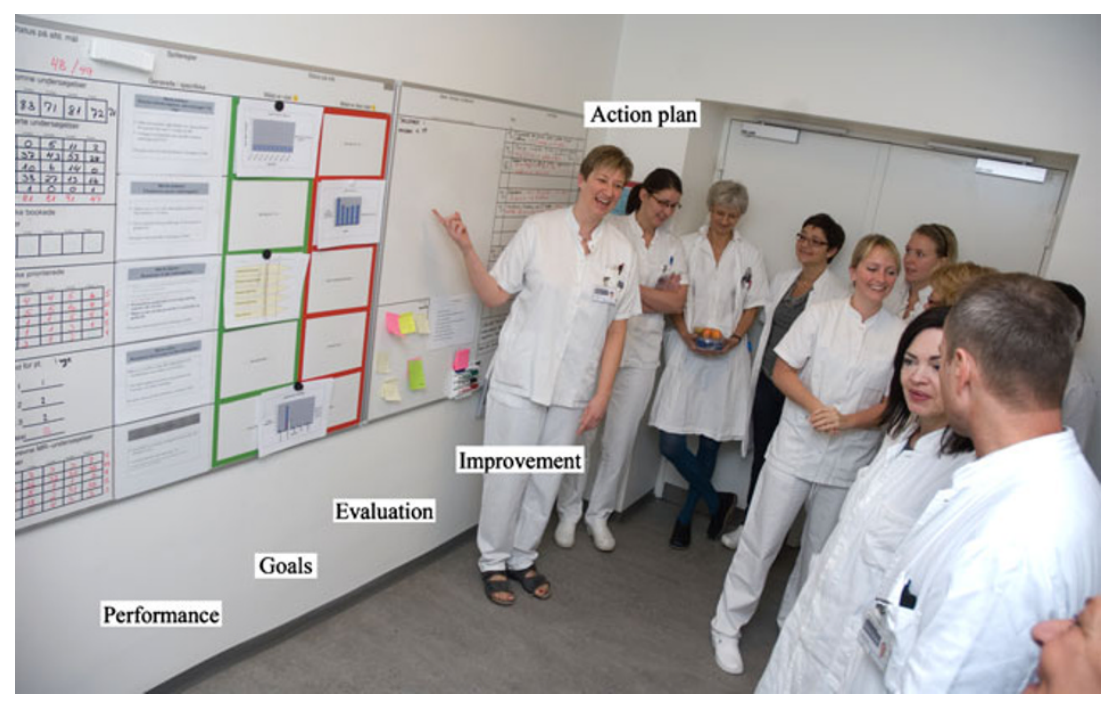

got involved, almost enthusiastically working with Lean, while some of the mid-level managers, i.e. the consultants and the superintendent radiographers, were more critical. Being the heads of the department, we were the first to be introduced to Lean. We had to learn to let go of part of the control and command. However, the mid-level managers seemed to have even more at risk. The normal top-down commands had changed with the Lean method. Suddenly the ideas of the youngest staff were just as valuable as the ideas of the senior consultants, and the proposals from the staff could even imply change in how the consultants had to work if it was of benefit to the patients and the workflow. It seemed that Lean created not just a change in culture but could even create a change in influence on the work and in personal authority, a challenge we had to take into consideration $[5,6]$.

Likewise, the Lean group could introduce changes in the different teams. The group's competences and authority, especially in respect of the middle management level in the department, had to be discussed. They gained inside knowledge from the different teams in the department, and it might be difficult for a superintendent of a team to accept improvement suggestions from the Lean group.

The values of mutual openness and respect are a must. It can be difficult to keep up good spirits in a big organisation with many individualists, to make sure that everyone is involved, educated and informed. Resistance to change is a well-known reaction [5, 6]. It is argued that members of an organisation seldom cooperate together, unless there is clear benefit for the individual. More often, people have different interests, which they try to realise in the daily life of the organisation. When people feel that a change threatens their interests they often resist the change, expressing weaknesses and disadvantages in the proposals without expressing that it is actually fear of the loss of personal authority and interests they feel. As Lean can change the culture, the ways of working and command, it is natural that it will create resistance, as we saw, especially at the middle management level.

The specific outcomes of working with Lean were decreasing waiting time for the patients, optimised workflow during the examinations, and an increased number of patients processed during a working day with only a marginal increase in costs. All in all, a higher productivity and more time gained for the staff for education, etc. In addition to the higher productivity, one of the benefits of Lean was a change in looking at how we work. Instead of doing things the way the employees used to, day after day, they started thinking of alternatives, showing more interest and giving thought to how they work.

Lean created more focus on the patients' needs. At weekly target meetings, we still discuss the service goals for the patients as well as focusing on whether the patients receive the services within the time limits defined by the department.

There is more time for training and development of the employees. The better production planning created the possibility of closing for non-acute production and arranging lessons or education days.

There is a better overview of the production, the problems and possible areas of development. Everyone was invited to participate in the dialogue and this has increased interdisciplinary cooperation.

The drawbacks were the concerns and uncertainty during the implementation of Lean. From a long-term perspective, Lean might reduce the creativity of, for example, the consultants, because of the standardisation in the work that Lean induces. However, up till now, this has not been the case. Only a few of the departments at the hospital 
introduced Lean at a time. Seen from the patients' perspective, the results would potentially be much higher if the whole hospital worked with Lean, optimising the handling of the patients in every aspect during their stay. At $\mathrm{OUH}$, Lean is still being implemented, however one department at a time.

\section{Conclusion}

Despite our own initial scepticism, as well as resistance from some of the staff, the Radiology Department was successful in introducing Lean. At times it was hard and gave reason for concerns and worries, but in the long run it was worth the effort. Lean provided higher productivity as well as extra time for education and meetings. Consequently, in spite of the industrial background of the Lean concept, it can successfully be used in a hospital radiology department.

Acknowledgements We thank Kirsten Pehrson for input into "Lean in the CT section".
Open Access This article is distributed under the terms of the Creative Commons Attribution Noncommercial License which permits any noncommercial use, distribution, and reproduction in any medium, provided the original author(s) and source are credited.

\section{References}

1. Taylor FW (1912) Scientific management. In: Pugh DS (ed) (1997) Organization theory: selected readings. Penguin Books, London, pp 275-295

2. Ohno T (1995) Toyota production system: beyond large scale production, Productivity Press, Portland

3. Womack JP, Jones DT (2003) Lean thinking: banish waste and create wealth in your corporation. Simon \& Schuster, New York

4. Mayo E (1949) Hawthorne and the Western Electric Company. In: Pugh DS (ed) (1997) Organization theory: selected readings. Penguin Books, London, pp 355-368

5. Krantz J (2001) Dilemmas of organizational change: a systems psychodynamic perspective. In: Gould L, Stapley LF, Stein M (eds) The systems psychodynamics of organizations. Karnac, New York London

6. Hirschorn L (2000) Changing structure is not enough: the moral meaning of organization design. In: Beer $\mathrm{M}$, Nohria $\mathrm{N}$ (eds) Breaking the code of change. Harvard Business School Press, Boston 